\title{
Effect of Cold-Water Immersion on Elbow Flexors Muscle Thickness After Resistance Training
}

\author{
Filipe Matos, ${ }^{1}$ Eduardo B. Neves, ${ }^{2}$ Claudio Rosa, ${ }^{1}$ Victor M. Reis, ${ }^{1}$ Francisco Saavedra, ${ }^{1}$ \\ Severiano Silva, ${ }^{3}$ Francisco Tavares, ${ }^{4}$ and José VilaÇa-Alves ${ }^{1}$ \\ ${ }^{1}$ Research Center for Sports, Health Sciences and Human Development, CIDESD, University of Trás-os-Montes and Alto Douro, \\ Vila Real, Portugal; ${ }^{2}$ Brazilian Army, Army Headquarters, Brasilia/DF, Brazil; ${ }^{3}$ Zootecnia Department, Trás-os-Montes and \\ Alto Douro University, Vila Real, Portugal; and ${ }^{4}$ Chiefs Super Rugby, Ruakura Research Center, Hamilton, New Zealand
}

\begin{abstract}
Matos, F, Neves, EB, Rosa, C, Reis, VM, Saavedra, F, Silva, S, Tavares, F, and Vilaça-Alves, J. Effect of cold-water immersion on elbow flexors muscle thickness after resistance training. J Strength Cond Res 32(3): 756-763, 2018-Cold-water immersion $(\mathrm{CWI})$ is commonly applied to speed up the recovery process after exercise. Muscle damage may induce a performance reduction and consequence of the intramuscular pressure induced by the muscular swelling. The aim of the study was to verify the CWI effects on muscle thickness (MT) behavior of the elbow flexors after a strength training (ST) protocol. Eleven men were submitted to an ST, performed in 2 different weeks. In one of the weeks, subjects experienced a passive recovery. In the other, subjects were submitted to a $\mathrm{CWI}\left(20\right.$ minutes at $5-10^{\circ} \mathrm{C}$ ). Ultrasound (US) images were taken before, after, as well as 24 , 48, and 72 hours after exercise, to evaluate the MT. Muscle thickness in both exercise arm (EA) and control arm (CA) was significantly higher 48 and 72 hours after exercise when subjects were submitted to a passive recovery compared with the CWI ( $p$ $=0.029, p=0.028, p=0.009$, and $p=0.001,48$ hours, 72 hours, EA, and CA, respectively). When each arm was analyzed with or without using CWI individually, significantly higher MT was observed in the EA with CWI: before exercise in relation to 72 hours after exercise $(p=0.042)$ and after exercise in relation to the other measurements $(p=0.003, p=0.003, p=0.038$, and $p$ $<0.0001$, before exercise and 24, 48, 72 hours after exercise, respectively). The evaluation of MT by US provides evidence that CWI after ST (and 24 hours after exercise) may reduce muscle swelling in the postexercise days when compared with a passive recovery. Seems to be a paradox between the uses of CWI for an acute reduction of muscle swelling.
\end{abstract}

Address correspondence to Filipe Matos, filipejosematos@gmail.com.

$32(3) / 756-763$

Journal of Strength and Conditioning Research

(C) 2017 National Strength and Conditioning Association

756

Journal of Strength and Conditioning Research
KEY WoRDS DOMS, swelling, cryotherapy, muscle damage

\section{INTRODUCTION}

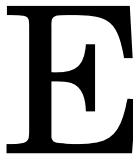

xercise, by itself, induces natural damage to the muscle-skeletal tissue, causing various responses, proportional to the duration, strength, and muscle mass recruited. The body's recovery to muscle damage caused by exercise will depend on the course of time, the severity of pain, and the level of muscular dysfunction $(6,16)$.

Athletes, coaches, and individuals exercising for recreation and practitioners working with clinical populations often seek ancillary strategies to enhance the muscular recovery process. In sports medicine, many postexercise recovery techniques have been investigated to find methods to reduce the secondary symptoms of the magnitude of damage caused by exercise and to accelerate the recovery process to help regular individuals and athletes to maintain the required workload during subsequent training sessions while reducing the risk of injury (34). Cryotherapy is one of the strategies, which is widely implemented to enhance recovery in athletes (e.g., Rugby ref) or individual's recreational training (31).

Several methods of cryotherapy are becoming increasingly popular as a tool to improve muscular recovery after exercise. In fact, the physiological responses to these methods are still poorly understood $(3,11)$. The literature has shown that the magnitude of tissue temperature change is correlated positively with cryotherapy methods that undergo a phase change (20) have a higher thermal gradient (38), long-term through time, (13) or are applied over a larger surface area (14).

Cold-water immersion (CWI), which involves immersing the body or body parts in water at temperatures below $15^{\circ} \mathrm{C}$ for periods of between 3 and 20 minutes, is commonly applied to speed up the muscular recovery process (11). However, despite the development of clinical guidelines, the literature is inconclusive regarding its applicability. There is no consensus on the effectiveness of these methods, continuing with ambiguous protocols, and without optimal application times $(3,27)$. 
Bleakley et al. (3) performed a systematic review aiming to understand the effectiveness of CWI protocols to prevent and treat the muscle-skeletal damage caused by exercise, published in The Cochrane Library. A total of 17 studies, published between 1998 and 2009, were included in this review. Among the 17 studies included in this review, there were only 5 that studied the effects of CWI after a strength training (ST) protocol $(7,9,17,30,39)$, and of these 5 studies, there was only one that compared the effects of CWI with a passive recovery (39).

Therefore, Bleakley et al. (3) concluded that there was no consensus regarding the effects of CWI protocols on muscular recovery because of various and poor methodologies applied. To the date of this writing, only 4 more studies involving RT and CWI protocols can be found (8,25-27).

The application of cryotherapy methods, as well as CWI, aims to attenuate secondary effects such as pain, discomfort, edema, and muscular dysfunction. These secondary effects are common after a resistance training program designed to achieve muscular hypertrophy and usually achieves a peak between 24 and 72 hours after exercise $(7,20)$. Delayed onset muscle soreness (DOMS) is the usual term used to define this pain and muscular dysfunction (6).

Delayed onset muscle soreness may induce a performance reduction, disturb the muscular position sense, and cause a decrease in reaction time (23). Usually, these secondary effects are consequences of intramuscular pressure, induced by muscular swelling, in response to the muscular aggression (2). The quantity of muscle mass volume can be assessed by the measure of muscle thickness (MT), which can be evaluated by ultrasonography (21).

Thus, we aimed to verify the CWI effects on muscle thickness behavior of the elbow flexors after a strength training protocol.

\section{Methods}

\section{Experimental Approach to the Problem}

The experimental trial was divided into 2 weeks, which differs only in the muscular recovery therapies applied. On both weeks, the subjects performed the same ST protocol for the exercise arm (EA). The other arm did not perform any ST and was used as a control arm (CA). The difference between the weeks was the recovery therapy applied: (a) CWI and (b) passive recovery.

At the first and second sessions, a 1-repetition maximum (1RM) test was applied (15) for biceps curl (with forearm supination) and biceps curl hammer (without forearm supination) to infer the workload for each subject. Then, 72 hours later, the $1 \mathrm{RM}$ re-test was performed to achieve reliable workload data.

\section{Subjects}

Before providing their written informed consent, all participants were informed of the requirements and potential risks of the studies. The experimental procedures adhered to the standards set by the latest revision of the Declaration of Helsinki (2013), for ethics in research with human beings. The study was approved by the Institutional Review Board of the University of Trás-os-Montes \& Alto Douro. This study was a randomized controlled trial; participants were randomly assigned to either CWI or passive recovery in the first experimental session and ranged between 18 to 33 years old (Table 1). Eleven active men $(n=11)$, who were familiar with the biceps curl exercise and had been ST 2-3 times a week for the previous 6 months, volunteered to participate in this study. Experimental procedures and risks were explained to the participants before they provided their informed consent to take part in the study. The inclusion criteria were male gender, Caucasian ethnicity, and apparently healthy. To define the inclusion criteria, the participants completed the Par-Q test questionnaires (ACSM, 2007), an anamnesis specifically designed according the requirements of assessment methods involved in this study. The characteristics of the participants in this study are described in Table 1.

\section{Procedures}

All trials started at $10 \mathrm{Am}$ The participants were informed to eat similar food during the experiment trials, asked to avoid consuming stimulants, alcohol, tobacco, antioxidants, and nutritional supplementation for 24 hours preceding all trials, and informed not to do any strength exercise for 48 hours before each trial.

Resistance Training Protocol. Subjects were submitted to the same ST protocol in both weeks, under the same conditions: 5 sets of biceps curl (with forearm supination) and hammer biceps curl (neutral forearm position), organized in Bi-set, at $70 \%$ of $1 \mathrm{RM}$ (22). The rest period established was 1 minute 30 seconds. The movement cadence was $60 \mathrm{~b} \cdot \mathrm{min}^{-1}$ monitored by a metronome (Dolphin Dp31g).

Recovery Therapy Protocol. The recovery therapy protocol applied on the 2 experimental weeks was different (Figure 1). In one of the weeks, subjects were exposed to a CWI, and cryotherapy protocol was applied. The EA was immersed for 20 minutes in a container filled with ice and water at a temperature between 5 and $10^{\circ} \mathrm{C}$, immediately after exercise and 24 hours after. As a matter of safety, the hand was not immersed to avoid the adverse effects of low temperatures to the body extremities (19).

The water in the container was monitored with a Thermocouple DFT-M-700 (Shinko Technos Co., Osaka, Japan).

TABLE 1. Descriptive characteristics of subjects.

\begin{tabular}{lcccr}
\hline & $n$ & $\min$ & $\max$ & Mean $\pm S D$ \\
\hline Age & 11 & 18 & 33 & $20.50 \pm 3.92$ \\
Body height $(\mathrm{cm})$ & 11 & 164 & 180 & $1.76 \pm 4.55$ \\
Body mass $(\mathrm{kg})$ & 11 & 60.6 & 78.0 & $70.4 \pm 3.69$ \\
Body fat $(\%)$ & 11 & 3.7 & 16.5 & $9.4 \pm 3.30$ \\
\hline
\end{tabular}




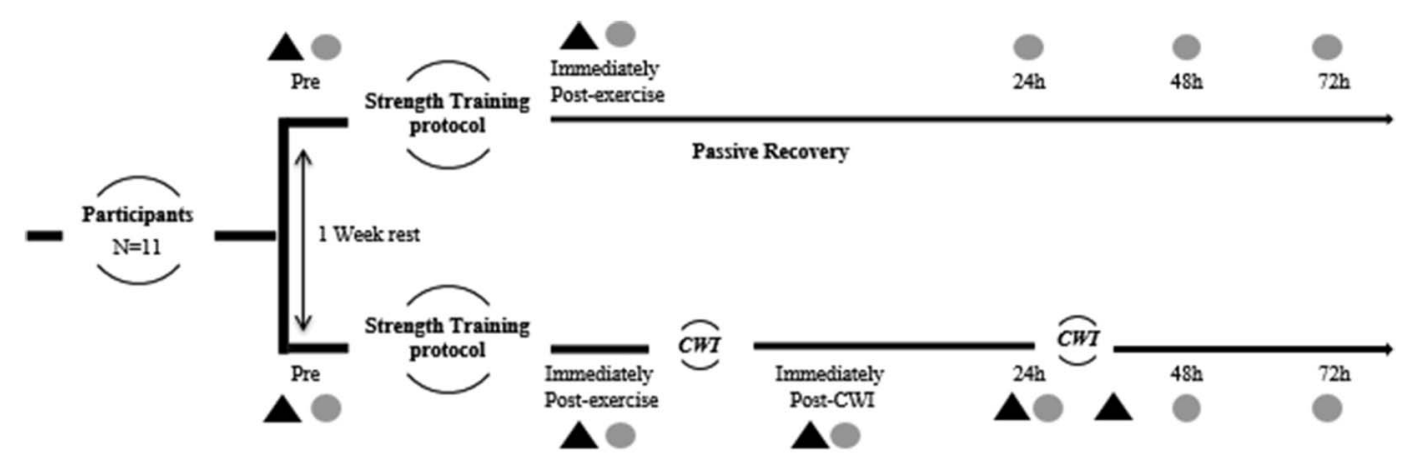
Ahermography
Ultrasonography

Figure 1. US image of the elbow flexors MT, considered as the distance between the interfaces of the muscle tissue, from the subcutaneous fat to the bone. $\mathrm{US}=$ ultrasound; MT $=$ muscle thickness.

The whole treatment process was carefully monitored for greater control of the water temperature and the subjects' safety. The addition of ice was made according to the requirements caused by the heat balance, to keep the temperature as constant as possible.

On the other week, there was no recovery therapy applied (passive recovery).

Muscle Thickness Assessment. Muscle thickness was obtained using an ultrasound (US) Aloka SSD 500V (Tokyo, Japan) with

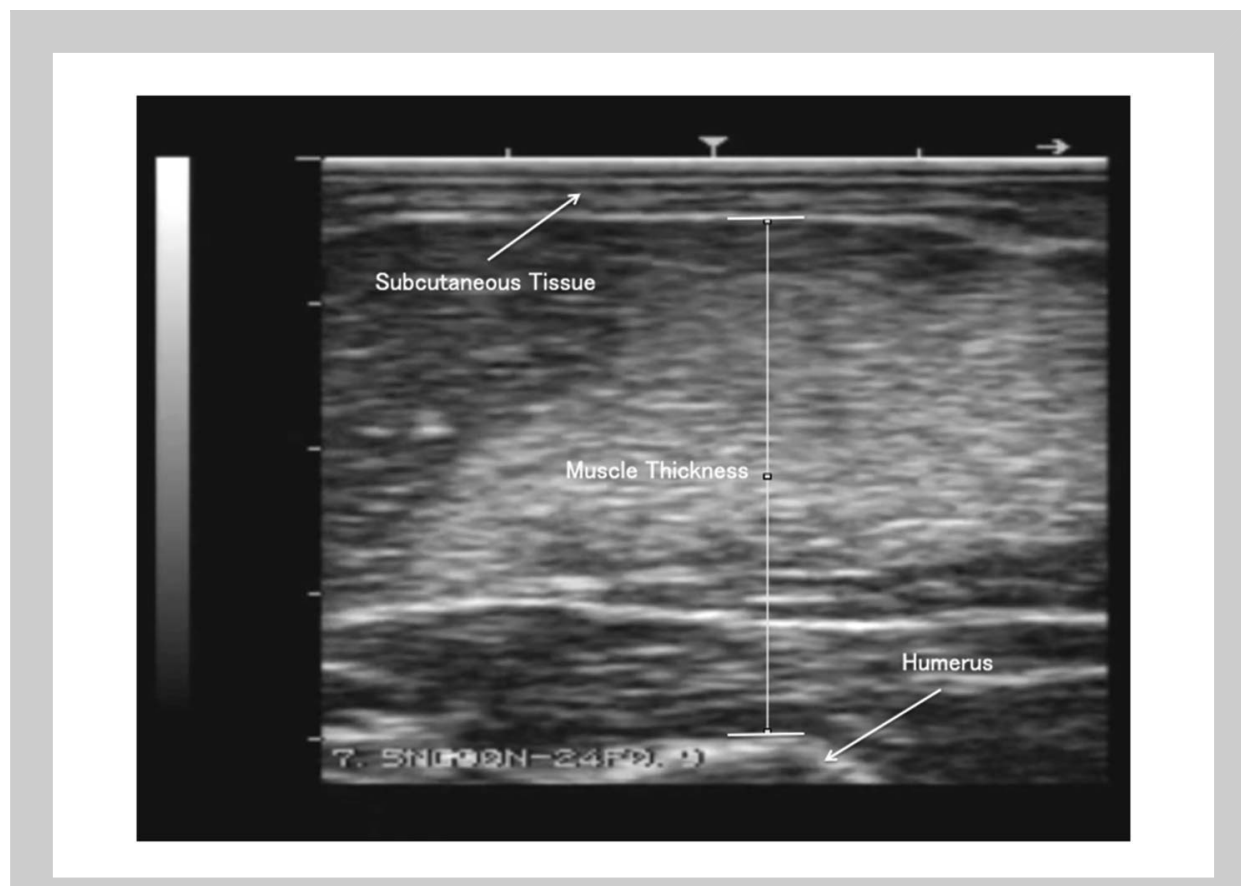

Figure 2. Ulrasound image accessed immediately post-exercise.

Journal of Strength and Conditioning Research an electronic linear transducer of $7.5 \mathrm{MHz}$ (UST-5512U-7.5, $38 \mathrm{~mm}$; Aloka, Noblesville, IN) wave frequency, used for a transverse scan. The ultrasound images were captured using a gain of $90 \mathrm{~dB}$ and a magnification, which allows for a depth of $42 \mathrm{~mm}$. Elbow flexors MT (biceps brachii; brachialis) were measured before and immediately after exercise, as well as 24, 48, and 72 hours after exercise. Ultrasound images were kept on video. To standardize the measurements, US images were acquired at $60 \%$ of the distance between the posterior ridge of the acromion and the olecranon of the arms, whereas the subject was seated with his arms relaxed on their respective sides (18). Elbow flexors MT was considered as the distance between the interfaces of the muscle tissue, from the subcutaneous tissue to the humerus bone (Figure 1). Ultrasound settings were kept unchanged throughout the image acquisitions (Figure 2).

Muscle thickness analysis was exported to a personal computer and analyzed using open-source ImageJ software version 1.37 (National institute of health, USA) as shown in Figure 1 (28).

\section{Skin Temperature}

Assessment. Thermal images were taken before and immediately after as well as 24,48 , 
TABLE 2. Mean $\pm S D$ of $\mathrm{T}_{\text {sk }}$ of the elbow flexors after the CWI protocol.*

\begin{tabular}{lcccc}
\hline CWI application & Pre-T & Post- $_{\text {sk }}$ & Post-pre & Post-pre (\%) \\
\hline Immediately postE & $34.60 \pm 0.62^{\circ} \mathrm{C}$ & $20.37 \pm 2.74^{\circ} \mathrm{C}$ & $14.23 \pm 2.78^{\circ} \mathrm{C}$ & 41.09 \\
$24-\mathrm{h}$ PostE & $35.15 \pm 1.08^{\circ} \mathrm{C}$ & $21.11 \pm 2.17^{\circ} \mathrm{C}$ & $14.04 \pm 1.99^{\circ} \mathrm{C}$ & 39.94 \\
\hline
\end{tabular}

${ }^{*} \mathrm{CWI}=$ cold-water immersion; PostE $=$ postexercise; $\mathrm{T}_{\mathrm{sk}}=$ skin temperature.

and 72 hours after exercise. Furthermore, thermal images were taken immediately after the CWI protocol. Images were acquired during morning (10:00 AM).

The skin surface temperature assessment was performed in a room with a conditioned environment within a range of 22- $24^{\circ} \mathrm{C}$, monitored by a digital thermometer (Elecs HTC2). Thermal images were captured using a Thermographic Camera Ti32 from Fluke Technologies (Mumbai, India).

The camera was positioned on a tripod $\pm 1 \mathrm{~m}$ above the ground and $\pm 1 \mathrm{~m}$ from the subject. There was a nonreflecting background behind the subject.

Thermal images were analyzed using regions of interest (ROI), which include the following: (a) 60\% of the distance between the posterior ridge of the acromion and the olecranon of the arm and (b) the xiphoid process as a reference of the central body temperature. These regions were accessed with the computer software SmartView Thermal Imaging Analysis (Fluke Technologies, Mumbai, India), which provided us with the average and mean temperatures from each analyzed ROI (19).

\section{Statistical Analyses}

The results are shown with the average $\pm S D$. Comparisons between arms (exercise and control) and between the immersion with and without the bath for each time point analyzed were determined using a $t$ test for independent variables. An ANOVA for repeated-measures analyzed was used with the following models: 5 moments (before exercise, after exercise, and 24, 48, and 72 hours after exercise) $\times 2$ sessions (with and without immersion bath) $\times 2$ arms (exercise and control). A Bonferroni post hoc test was used to identify differences between the moments, sessions, and arms. All data analyzed were tested for assumptions of normality, homogeneity, and sphericity with the shaphirowilk, lavene, and Mauchly tests, respectively. The level of significance was established at 5\%. The statistical analyses were conducted using SPSS 22.0 (SPSS, Inc., Chicago, IL, USA).

\section{Results}

On average, subjects were submitted to a CWI with a temperature of $8.3 \pm 0.76$. Table 2 shows the skin temperature reductions caused by the CWI in the elbow flexors of the arm submitted to the RT protocol.

When CWI was applied immediately after exercise, we can observe $\mathrm{T}_{\text {sk }}$ reductions around $14.00^{\circ} \mathrm{C}( \pm 2.78)$, in the elbow flexors ROI. Similarly, when CWI was applied 24 hours after exercise, we could observe $\mathrm{T}_{\text {sk }}$ reductions of $13.90^{\circ} \mathrm{C}( \pm 1.99)$.

TABLE 3. Mean $\pm S D$ of muscular thickness (millimeters) in exercise and control arms with (CWI) or without (WCWI) use immersion bath.*

\begin{tabular}{|c|c|c|c|c|}
\hline & \multicolumn{2}{|c|}{ Exercise arm } & \multicolumn{2}{|c|}{ Control arm } \\
\hline & CWI & WCWI & CWI & WCWI \\
\hline Pre-exercise & $26.72 \pm 4.93$ & $26.21 \pm 4.25$ & $24.98 \pm 5.04$ & $25.42 \pm 4.87$ \\
\hline Postexercise & $31.06 \pm 5.18 \S$ & $33.51 \pm 4.59 \S$ & $25.43 \pm 3.76 \ddagger$ & $26.47 \pm 5.50 \ddagger$ \\
\hline 24-h PostE & $26.78 \pm 3.939$ & $30.50 \pm 5.57 \S$ & $25.30 \pm 3.04$ & $26.11 \pm 5.12$ \\
\hline 48-h PostE & $26.84 \pm 4.17 \rrbracket$ & $31.90 \pm 4.08+\S$ & $23.66 \pm 3.72$ & $28.04 \pm 4.90 \dagger \S$ \\
\hline 72-h PostE & $23.83 \pm 4.98 \S \rrbracket \#$ & $31.81 \pm 5.04 \uparrow \S$ & $23.47 \pm 4.57$ & $28.32 \pm 5.04 \uparrow \S \|$ \\
\hline
\end{tabular}

${ }^{*} \mathrm{CWI}=$ cold-water immersion; PostE $=$ postexercise.

$\dagger p \leq 0.05$ between the use and not use of immersion bath.

$\pm p \leq 0.05$ between control and exercise arms.

$\$ p \leq 0.05$ in relation to the pre-exercise moment.

$\| p=0.037$ in relation to 24 -hour postexercise moment.

I $p \leq 0.05$ in relation to the postexercise moment.

$\# p=0.031$ in relation to the 48 -hour postexercise moment. 
Regarding muscle thickness, a time effect was observed $\left(F_{(4,160)}=7.030 ; p<0.0001 ; \mu_{\mathrm{p}}^{2}=0.149\right)$, an interaction of time $\times$ with or without the use of CWI $\left(F_{(4,160)}=12.329\right.$; $\left.p<0.0001 ; \mu_{\mathrm{p}}^{2}=0.236\right)$, an interaction of time $\times \mathrm{EA}$ or $\mathrm{CA}$ $\left(F_{(4,160)}=4.172 ; p=0.005 ; \mu_{\mathrm{p}}^{2}=0.094\right)$, with or without the use of CWI effect $\left(F_{(1,40)}=5.319 ; p=0.026 ; \mu_{\mathrm{p}}^{2}=0.117\right)$, and an arm effect $\left(F_{(1,40)}=5.996 ; p=0.019 ; \mu_{\mathrm{p}}^{2}=0.130\right)$. No significant interaction was observed between time, with or without the use of CWI, and the EA or CA.

As shown in the Table 3, the muscular thicknesses in the EA and CA were significantly higher at 48 and 72 hours after exercise without the use of CWI $(p=0.029, p=0.028$ and $p=0.009, p=0.001$, for the 48 hours, 72 hours, EA and CA, respectively). When the $\mathrm{EA}$ and $\mathrm{CA}$ were compared between with or without the use of CWI, in the moment after exercise, the muscular thickness was significantly higher in the EA in both situations $(p=0.030$ and $p=0.008$, with and without use of CWI, respectively).

When each arm with or without the use of CWI was analyzed individually, a significantly higher muscular thickness was observed in the EA with the use of CWI (Table 1): in the pre-exercise moment in relation to the 72 hours after exercise moment $(p=0.042)$ and in the postexercise moment compared with the other moments $(p=0.003$, $p=0.003, p=0.038$, and $p<0.0001$, pre-exercise and 24, 48 , and 72 hours after exercise, respectively). Also, in the EA but without the use of CWI, significantly fewer values of muscular thickness were observed in the moment before exercise compared with the other moments $(p<0.0001$, $p=0.001, p<0.0001$, and $p=0.002$, after and 24,48 , and 72 hours after exercise, respectively).

\section{Discussion}

Cold-water immersion has been applied as a muscular recovery therapy. However, there are multiple opinions about its efficacy when applied after an exercise protocol in the literature.

When applying any method of cryotherapy, monitoring $\mathrm{T}_{\text {sk }}$ is of particular importance regarding caution and safety of the participants submitted to low temperatures but also because to achieve physiological responses from the application of cryotherapy, the methods have to be able to reduce the $\mathrm{T}_{\mathrm{sk}}$ by at least $10-15^{\circ} \mathrm{C}(19,29)$. Also, Hardaker et al. (10) show that there is a strong relationship between $T_{\text {sk }}$ and intramuscular temperature after $15 \mathrm{mi}-$ nutes of clinical cryotherapy application. As such, $\mathrm{T}_{\text {sk }}$ can be considered a highly relevant indicator of intramuscular temperature $3-\mathrm{cm}$ subadipose. We monitored $\mathrm{T}_{\mathrm{sk}}$ during the entire process.

In this study, we observe $\mathrm{T}_{\text {sk }}$ reductions of $14.00^{\circ} \mathrm{C}( \pm 2.78)$ when CWI was applied immediately after exercise. Similarly, when cryotherapy was applied 24 hours after exercise, a $\mathrm{T}_{\text {sk }}$ reduction of $13.90^{\circ} \mathrm{C}( \pm 1.99)$ was observed. However, even with these parameters documented, the physiological aspects from CWI application are not fully understood.
Delayed onset muscle soreness is a well-documented phenomenon, often occurring as the result of unaccustomed or high-intensity exercise. Associated symptoms include muscle shortening, increased passive stiffness, swelling, decreases in strength and power, localized soreness, and disturbed proprioception. Depending on the specific nature of the exercise, the stress induced can be predominantly metabolic, mechanical, or both (32). In brief, ST-inducing primary metabolic stress in active skeletal muscles involves a high rate of aerobic and anaerobic energy transformation (4) and heat generation (1). Both contribute to an increase in the generation of reactive oxygen species (ROS). Reactive oxygen species is highly reactive and can denature proteins, nucleic acids, and lipids, which destabilize muscle cell structures including the sarcolemma and structures of the excitation-contraction coupling system (24). Damage to the excitation-contraction coupling system alters contraction kinetics, thereby reducing force-generating capacity and athletic performance, whereas disruption of the sarcolemma makes the muscle fiber more permeable (5).

The sustained high transformation of energy to support repeated contractions and increased intramuscular pressure imposed by hyperemia (40) can also impose mild hypoxic stress on the muscle fibers, promoting an accumulation of metabolites (4). The accumulation of metabolites within the cell caused by a high metabolic rate increases the osmolality of the cell. Paired with increased permeability, the potential for cell swelling is enhanced (2).

Coupled with the damage by ROS and muscle fiber swelling, an exercise-induced inflammatory response is initiated. Although inflammation is required for the resolution of any muscle fiber damage resulting from the exercise insult, if excessive or unabated, the phagocytic activity of neutrophils and macrophages contribute to secondary muscle damage. Secondary muscle damage, damage incurred by the inflammatory response to exercise and not the exercise bout per se, compounds the soreness and reduction in force-generating capacity experienced in the hours and days after a high-intensity exercise bout (33).

Loss of structural integrity of the sarcolemma and contractile system is directly induced by the strain experienced during contractions in response to mechanical stress. Sarcolemma disruption enhances cell permeability and swelling, whereas disruption to the excitation-contraction coupling system impairs force-producing capability and both contribute to soreness and reduced function (2).

In this study, we have tried to verify the CWI effects on muscle thickness behavior of the elbow flexors after a strength training protocol.

When both the EA and CA were compared with or without the application of the CWI protocol, in the postexercise period, the MT was significantly higher in the EA in both situations $(p=0.030$ and $p=0.008$, with and without CWI use, respectively), which was expected and has been documented after RT training and an acute immunologic 
response to the aggression induced in the active musculature, which exponentially increases the muscular swelling $(2,5,33)$.

Also, the MT in both EA and CA were significantly higher 48 and 72 hours after exercise when the subjects experimented a passive recovery compared with the CWI protocol application $(p=0.029, p=0.028$ and $p=0.009, p=0.001,48$ hours, 72 hours and EA, CA, respectively).

When each arm was analyzed individually, with or without CWI application, significantly higher muscular thickness was observed in the EA with CWI application: in the pre-exercise period in relation to 72 hours after exercise $(p=0.042)$ and in the postexercise period in relation to the other moments $(p=0.003, p=0.003, p=0.038$ and $p$ $<0.0001$, before exercise and $24,48,72$ hours after exercise, respectively). However, in the EA, but without CWI application, significantly fewer values of muscular thickness were observed before exercise in relation to the other time points $(p<0.0001, p=0.001, p<0.0001$, and $p=0.002$, after and $24,48,72$ hours after exercise, respectively). These data showed that when CWI was applied immediately after and 24 hours after hypertrophy RT protocol, the MT decreases significantly more in the recovery period (24-72 hours after exercise) when compared with passive recovery.

Despite the methodological differences, these data are similar to the findings obtained by Vaile et al. (35) who examined the effect of the 3 cryotherapy interventions (CWI, hot-water immersion, and contrast water therapy) in comparison with a passive rest recovery after a controlled ST protocol. Identical durations of recovery, water exposure, and temperatures were maintained. The authors observed showed that CWI and contrast water therapy, but not hotwater immersion, significantly reduced the degree of postexercise swelling when compared with active recovery (35).

It was the aim of this study to measure the swelling behavior from ST protocol after CWI. The reduced blood flow to the injured area decreases the permeability of blood vessels, which may be a major factor in reducing the inflow of material to the injured muscle cell. In this study, when each arm with or without the use of CWI was analyzed individually, significantly higher muscular thickness was observed in the EA with the use of CWI: in the pre-exercise period in relation to 72 hours after exercise $(p=0.042)$ and in the postexercise moment in relation to the other moments $(p=0.003, p=0.003, p=0.038$, and $p<$ 0.0001 , before exercise and $24,48,72$ hours after exercise, respectively). The edema induced by exercise seems to be biphasic, so that the initial increase in cell volume occurs acutely during the first 2 hours after exercise, because of the changes in osmotic pressure (accumulation of metabolites and extracellular proteins resulting from mechanical damage), whereas the subacute increase, between 24 and 96 hours after exercise, is due to secondary inflammatory damage.

The decrease in blood flow coupled with the decrease in cell metabolism may provide a lower consumption of oxygen to the cell, surviving for a longer period of ischemia and reducing the metabolic stress experienced by the muscle cell after exercise, helping to reduce the disparity between the $\mathrm{O}_{2}$ availability and consumption. The function of the mitochondrial respiratory chain significantly contributes to ROS production by muscle cells. Thus, reducing the rate of mitochondrial energy production by the decreased temperature can be expected to result in more limited damage by ROS $(12,26,36)$. In addition, compressive forces commonly combined with cold (i.e., hydrostatic forces of water) structurally limit swelling and fluid accumulation, while facilitating the removal of wastes and increasing central blood volume (37).

Recently, Roberts et al. (26) added some new data that were in accordance with this theory. The authors examined the effects of CWI and active recovery on cardiac dynamics, muscle hemodynamic, tissue temperature, and strength after resistance exercise. On separate days, 10 men performed resistance exercise, followed by 10 minutes of CWI at $10^{\circ}$ $\mathrm{C}$ or 10 minutes of low-intensity cycling. Cold-water immersion reduced hemodynamic and tissue temperature and helped to maintain muscle strength after resistance exercise.

It seems evident that CWI can reduce muscle swelling in the postexercise recovery days. However, if the swelling muscle is the main cause of loss of performance, loss of function, reduced capacity to generate power, and induces muscular pain, it is expected that the reduction of muscular swelling will attenuate these side effects. In this study, the effects of CWI on muscle function, pain, or capacity to generate strength were not directly investigated, but a few studies have recently been documented with that aim, which gives support to our investigation. Vaile et al. (35) stated that when a specific movement (squat jump) was performed requiring dynamic power, CWI enhanced the recovery of both isometric force production and squat jump performance, compared with an active recovery.

Also, Roberts et al. (26) showed another new and important finding in their study; CWI prevented a decrease in maximal isometric strength after resistance exercise. By contrast, strength remained below pre-exercise values for at least 40 minutes after active recovery. To supplement these data, Roberts et al. (25) tried to understand how CWI influences the recovery of maximal and submaximal muscle function after high-intensity resistance exercise in another investigation. The authors suggest that CWI after resistance exercises allows athletes to complete more work during subsequent training sessions. Compared with active recovery, CWI did not alter the recovery of maximal strength or countermovement jump performance. However, it did enhance the recovery of submaximal muscle function during a high-intensity resistance exercise test.

These findings add to existing knowledge of the performance benefits and physiological effects of CWI after exercise; there is evidence that CWI after an RT protocol can reduce muscle swelling, enhance submaximal muscle function, and prevent any decrease in maximal isometric strength. 
These are important findings regarding CWI effects, especially for athletes, because recovery between training sessions is a highly relevant factor in a long-term adaptation to unaccustomed exercise and in the performance of the following sessions. The recovery time is particularly important for athletes who are often subjected to daily and weekly training sessions, which have yet to be combined with competitions.

Muscle inflammation as well as the metabolic product and the muscle swelling play an important role for the resolution of any muscle fiber damage resulting from the exercise insult and for those with perspective muscle hypertrophy. Thus, a paradox between the use of CWI for acute reduction in muscle swelling to facilitate recovery and the potential negative effects caused by blunting the stress response may exist.

It is important to note some limitations of the study. The small sample size used limits the extrapolation of the findings, nevertheless, similar sample sizes were used in previous research investigating the physiological effects of CWI $(12,20)$. In addition, in this study, a circulator was not used in the CWI, which explains the large $\left(5^{\circ}\right)$ variation in the water temperature during the protocol.

\section{Pratical Applications}

Individuals and athletes, who use resistance training to improve athletic performance, recover from injury, or maintain their health, should therefore reconsider using CWI as an adjuvant to their training; although to recover muscle function and performance, CWI seems to be a useful method.

\section{ACKnowledgments}

NanoSTIMA: Macro-to-Nano Human Sensing: Towards Integrated Multimodal Health Monitoring and Analytics of operation NORTE-01-0145-FEDER-000016, co-financed by the European Regional Development Fund (ERDF) through the NORTE 2020 (North Regional Operational Program 2014/2020). The authors have no conflicts of interest to disclose.

\section{REFERENCES}

1. Arbogast, $\mathrm{S}$ and Reid, MB. Oxidant activity in skeletal muscle fibers is influenced by temperature, $\mathrm{CO}_{2}$ level, and muscle-derived nitric oxide. Am J Physiol Regul Integr Comp Physiol 287: R698-R705, 2004.

2. Armstrong, RB. Initial events in exercise-induced muscular injury. Med Sci Sports Exerc 22: 429-435, 1990.

3. Bleakley, C, McDonough, S, Gardner, E, Baxter, GD, Hopkins, JT, and Davison, GW. Cold-water immersion (cryotherapy) for preventing and treating muscle soreness after exercise. In: The Cochrane Collaboration, Editor. Cochrane Database of Systematic Reviezes [Internet]. Chichester, UK: John Wiley \& Sons, Ltd, 2012. Available at: http://doi.wiley.com/10.1002/14651858.CD008262. pub2. Accessed: July1, 2015.

4. Clanton, TL. Hypoxia-induced reactive oxygen species formation in skeletal muscle. J Appl Physiol 102: 2379-2388, 2007.

5. Clarkson, PM and Sayers, SP. Etiology of exercise-induced muscle damage. Can J Appl Physiol 24: 234-248, 1999.
6. Cleak, MJ and Eston, RG. Delayed onset muscle soreness: Mechanisms and management. J Sports Sci 10: 325-341, 1992.

7. Eston, R and Peters, D. Effects of cold water immersion on the symptoms of exercise-induced muscle damage. J Sports Sci 17: 231238, 1999.

8. Fröhlich, M, Faude, O, Klein, M, Pieter, A, Emrich, E, and Meyer, T. Strength training adaptations after cold-water immersion. J Strength Cond Res 28: 2628-2633, 2014.

9. Goodall, S and Howatson, G. The effects of multiple cold water immersions on indices of muscle damage. J Sports Sci Med 7: 235241, 2008.

10. Hardaker, N, Moss, AD, Richards, J, Jarvis, S, McEwan, I, Selfe, J. Relationship between intramuscular temperature, and skin surface. Temperature as measured by thermal imaging camera. Thermol Int 17: 45-50, 2007.

11. Hohenauer, E, Taeymans, J, Baeyens, JP, Clarys, P, and Clijsen, R. The effect of post-exercise cryotherapy on recovery characteristics: A systematic review and meta-analysis. Alway se, editor. PLoS One 10: $\mathrm{e} 0139028,2015$

12. Ihsan, M, Watson, G, Lipski, M, and Abbiss, CR. Influence Post Exercise Cooling Muscle Oxygenation Blood Volume Changes. Med Sci Sports Exerc 45: 876-882, 2013.

13. Ingram, J, Dawson, B, Goodman, C, Wallman, K, and Beilby, J. Effect of water immersion methods on post-exercise recovery from simulated team sport exercise. J Sci Med Sport 12: 417-421, 2009.

14. Janwantanakul, P. The effect of quantity of ice and size of contact area on ice pack/skin interface temperature. Physiotherapy 95: 120 125, 2009.

15. Kraemer, W and Fry, A. Development and evaluation of methology. Phyological assessment of human fitness. Hum Kinet 15: 115-138, 1995.

16. Kraemer, WJ and Ratamess, NA. Hormonal responses and adaptations to resistance exercise and training. Sports Med 35: 339 361, 2012.

17. Kuligowski, LA, Lephart, SM, Giannantonio, FP, and Blanc, RO. Effect of whirlpool therapy on the signs and symptoms of delayedonset muscle soreness. J Athl Train 33: 222, 1998.

18. Lima, KMM, Carneiro, SP, Alves Dde, S, Peixinho, CC, and de Oliveira, LF. Assessment of muscle architecture of the biceps femoris and vastus lateralis by ultrasound after a chronic stretching program. Clin J Sport Med 25: 55-60, 2015.

19. Matos, F, Neves, EB, Norte, M, Rosa, C, Reis, VM, and Vilaça-Alves, $\mathrm{J}$. The use of thermal imaging to monitoring skin temperature during cryotherapy: A systematic review. Infrared Phys Technol 73: 194-203, 2015.

20. Merrick, MA, Jutte, LS, and Smith, ME. Cold modalities with different thermodynamic properties produce different surface and intramuscular temperatures. J Athl Train 38: 28-33, 2003.

21. Neves, EB, Moreira, TR, Lemos, R, Vilaça-Alves, J, Rosa, C, and Reis, VM. Using skin temperature and muscle thickness to assess muscle response to strength training. Rev Bras Med Esporte 21: 350354, 2015.

22. Neves, EB, Vilaça-Alves, J, Moreira, TR, Lemos, R, and Reis, VM. The thermal response of biceps Brachii to strength training. Gazzetta Med Ital Arch Sci Mediche 175: 391-399, 2016.

23. Paschalis, V, Nikolaidis, MG, Giakas, G, Jamurtas, AZ, Owolabi, EO, and Koutedakis, Y. Position sense and reaction angle after eccentric exercise: The repeated bout effect. Eur J Appl Physiol 103: 9-18, 2008

24. Powers, SK and Jackson, MJ. Exercise-induced oxidative stress: Cellular mechanisms and impact on muscle force production Physiol Rev 88: 1243-1276, 2008.

25. Roberts, LA, Nosaka, K, Coombes, JS, and Peake, JM. Cold water immersion enhances recovery of submaximal muscle function after resistance exercise. AJP Regul Integr Comp Physiol 307: R998-R1008, 2014. 
26. Roberts, LA, Muthalib, M, Stanley, J, Lichtwark, G, Nosaka, K, Coombes, JS, Peake, JM. Effects of cold water immersion and active recovery on hemodynamics and recovery of muscle strength following resistance exercise. Am J Physiol Regul Integr Comp Physiol 309: R389-R398, 2015.

27. Roberts, LA, Raastad, T, Markworth, JF, Figueiredo, VC, Egner, IM, Shield, A, Cameron-Smith, D, Coombes, JS, Peake, JM. Post-exercise cold water immersion attenuates acute anabolic signalling and longterm adaptations in muscle to strength training: Cold water immersion and adaptation to strength training. J Physiol 593: 4285-4301, 2015.

28. Schneider, CA, Rasband, WS, and Eliceiri, KW. NIH image to ImageJ: 25 years of image analysis. Nat Meth 9: 671-675, 2012.

29. Selfe, J, Hardaker, N, Thewlis, D, and Karki, A. An accurate and reliable method of thermal data analysis in thermal imaging of the anterior knee for use in cryotherapy research. Arch Phys Med Rehabil 87: $1630-1635,2006$

30. Sellwood, KL, Brukner, P, Williams, D, Nicol, A, and Hinman, R. Ice-water immersion and delayed-onset muscle soreness: A randomised controlled trial. Br J Sports Med 41: 392-397, 2007.

31. Tavares, F, Smith, TB, and Driller, M. Fatigue and recovery in rugby: A review. Sports Med 31: 1515-1530, 2017.

32. Tee, JC, Bosch, AN, and Lambert, MI. Metabolic consequences of exercise-induced muscle damage. Sports Med 37: 827-836, 2012.

33. Tidball, JG. Inflammatory processes in muscle injury and repair. $A m$ J Physiol Regul Integr Comp Physiol 288: R345-R353, 2005.
34. Torres, R, Ribeiro, F, Alberto Duarte, J, and Cabri, JMH. Evidence of the physiotherapeutic interventions used currently after exerciseinduced muscle damage: Systematic review and meta-analysis. Phys Ther Sport 13: 101-114, 2012.

35. Vaile, J, Halson, S, Gill, N, and Dawson, B. Effect of hydrotherapy on the signs and symptoms of delayed onset muscle soreness. Eur $J$ Appl Physiol 102: 447-455, 2008.

36. White, GE and Wells, GD. Cold-water immersion and other forms of cryotherapy: Physiological changes potentially affecting recovery from high-intensity exercise. Extreme Physiol Med 2: 1-11, 2013.

37. Wilcock, IM, Cronin, JB, and Hing, WA. Physiol response water immersion: A method sport recovery? Sports Med Auckl NZ 36: 747$765,2006$.

38. Yanagisawa, $\mathrm{O}$ and Fukubayashi, T. Diffusion-weighted magnetic resonance imaging reveals the effects of different cooling temperatures on the diffusion of water molecules and perfusion within human skeletal muscle. Clin Radiol 65: 874-880, 2010.

39. Yanagisawa, O, Niitsu, M, Yoshioka, H, Goto, K, Kudo, H, and Itai, Y. The use of magnetic resonance imaging to evaluate the effects of cooling on skeletal muscle after strenuous exercise. Eur J Appl Physiol 89: 53-62, 2003.

40. Zhang, $Q$ and Styf, J. Abnormally elevated intramuscular pressure impairs muscle blood flow at rest after exercise. Scand J Med Sci Sports 14: 215-220, 2004. 